\title{
Multiple-breath inert gas washout and spirometry versus structural lung disease in cystic fibrosis
}

\author{
P M Gustafsson, ${ }^{1}$ P A De Jong, ${ }^{2,3,4}$ H A W M Tiddens, ${ }^{2}$ A Lindblad ${ }^{1}$
}

\section{See related editorial page 96 \\ - Additional data are published online only at http://thorax.bmj. com/content/vol63/issue2}

${ }^{1}$ Queens Silvia Children's Hospital and Department of Pediatrics, The Sahlgrenska Academy at Göteborg, Sweden;

${ }^{2}$ Erasmus Medical Center-

Sophia Children's Hospital, Department of Pediatric

Pulmonology and Allergology, Rotterdam, The Netherlands;

${ }^{3}$ Meander Medical Center, Department of Radiology, Amersfoort, The Netherlands;

${ }^{4}$ University Medical Center

Utrecht, Department of

Radiology, Utrecht, The

Netherlands

Correspondence to:

Dr P M Gustafsson, Department of Paediatric Clinical Physiology, Queen Silvia Childrens Hospital, S-416 85 Göteborg, Sweden; per.gustafsson@vgregion.se

Received 18 January 2007 Accepted 19 July 2007

Published Online First

3 August 2007

\section{ABSTRACT}

Background: A sensitive and valid non-invasive marker of early cystic fibrosis (CF) lung disease is sought. The lung clearance index (LCl) from multiple-breath washout (MBW) is known to detect abnormal lung function more readily than spirometry in children and teenagers with $C F$, but its relationship to structural lung abnormalities is unknown. A study was undertaken to determine the agreements between $\mathrm{LCl}$ and spirometry, respectively, with structural lung disease as measured by highresolution computed tomography (HRCT) in children and teenagers with CF.

Methods: A retrospective study was performed in 44 consecutive patients with CF aged 5-19 years (mean 12 years). At an annual check-up inspiratory and expiratory HRCT scans, LCl and spirometric parameters (forced expiratory volume in $1 \mathrm{~s}\left(\mathrm{FEV}_{1}\right)$ and maximal expiratory flow when $75 \%$ of forced vital capacity was expired $\left(\mathrm{FEF}_{75}\right)$ ) were recorded. Abnormal structure was defined as a composite HRCT score of $>5 \%$, the presence of bronchiectasis or air trapping $>30 \%$. Abnormal lung function was defined as $\mathrm{LCl}$ above the predicted mean +1.96 residual standard deviations (RSD), or $\mathrm{FEV}_{1}$ or $\mathrm{FEF}_{75}$ below the predicted mean $-1.96 \mathrm{RSD}$. Sensitivity/specificity assessments and correlation analyses were done.

Results: The sensitivity to detect abnormal lung structure was $85-94 \%$ for LCl, $19-26 \%$ for $\mathrm{FEV}_{1}$ and $62-75 \%$ for $\mathrm{FEF}_{75}$. Specificity was $43-65 \%$ for $\mathrm{LCl}, 89-100 \%$ for $\mathrm{FEV}_{1}$ and $75-88 \%$ for $\mathrm{FEF}_{75}$. LCl correlated better with HRCT scores (Rs +0.85) than $\mathrm{FEV}_{1}(-0.62)$ or $\mathrm{FEF}_{75}(-0.66)$. Conclusions: $\mathrm{LCl}$ is a more sensitive indicator than $\mathrm{FEV}_{1}$ or $\mathrm{FEF}_{75}$ for detecting structural lung disease in $\mathrm{CF}$, and a normal LCl almost excludes HRCT abnormalities. The finding of an abnormal $\mathrm{LCl}$ in some patients with normal HRCT scans suggests that $\mathrm{LCl}$ may be even more sensitive than HRCT scanning for detecting lung involvement in CF.

Cystic fibrosis (CF) lung disease is characterised by persistent infection and inflammation from early in life, ${ }^{1}$ leading to chronic airway disease and a progressive decline in lung function. Airway abnormalities are thought to start in the peripheral airways, but eventually lead also to the destruction of the larger airways (bronchiectasis). Over recent decades the median age of survival of patients with CF has improved dramatically to 35 years in 2004, with most morbidity and mortality caused by CF lung disease. ${ }^{1}$ Intensive clinical monitoring of the airway disease and early intervention are needed to delay lung disease progression..$^{2-5}$ Sensitive markers of early lung involvement and sensitive methods to monitor progression of CF lung disease are therefore sought. ${ }^{126}$
Spirometry (forced expiratory volume in $1 \mathrm{~s}$ $\left.\left(\mathrm{FEV}_{1}\right)\right)$ is still the most widely used method for clinical monitoring of CF lung disease, and it is believed to be a good predictor of outcome in patients with moderate to severe CF lung disease. ${ }^{7-}$ ${ }_{9}$ Over the last two decades there has been a shift towards closer monitoring and more aggressive treatment of early CF lung disease. As a consequence, two major disadvantages of the use of $\mathrm{FEV}_{1}$ in children with CF have become apparent. First, many school-age children with CF now have $\mathrm{FEV}_{1}$ within the normal range ${ }^{10}$ or show a slow rate of progression, ${ }^{20-12}$ even though they probably have lung disease that progresses faster than is reflected in spirometric measurements. Apparently $\mathrm{FEV}_{1}$ is not very sensitive to early CF lung disease. ${ }^{13-15}$ Second, reliable forced expiratory manoeuvres are difficult to obtain in children under the age of 5 years, with testing in the infant and preschool age groups being largely confined to specialist laboratories. ${ }^{16-18}$ As interest in monitoring younger patients increases, so does the need for alternative more sensitive measures of CF lung disease that can be obtained in children of all ages.

High-resolution CT (HRCT) scanning of the chest has repeatedly been shown to be more sensitive than $\mathrm{FEV}_{1}$ both for early detection of CF lung disease $e^{19-22}$ and for following disease progression. ${ }^{23-25}$ Bronchiectasis, the hallmark of CF lung disease, can be identified early in the course of CF on HRCT scanning ${ }^{20}$ and is the HRCT abnormality that is most sensitive to disease progression. ${ }^{63-24}$ HRCT is the current gold standard to evaluate bronchiectasis. ${ }^{26}$ Air trappingreflecting small airways disease-is another important feature of CF lung disease that can be demonstrated with expiratory HRCT scans. ${ }^{27} 28$ Furthermore, mucus plugging and airway wall thickening can be seen on inspiratory scans in CF. ${ }^{29-34}$ The use of CT scanning in CF is, however, restricted to examinations with a relatively long interval of 1-2 years. Despite a substantial reduction in the dose of radiation used in CT examinations of the chest in the last decade, its use should be limited to the absolute minimum. ${ }^{35-37}$ Hence, CT scanning is not a feasible method for monitoring CF lung disease over short time intervals. In addition, routine CT scanning in young children is troublesome because sedation and a technically demanding procedure are required to obtain high quality images. ${ }^{38}$ In addition, young children are thought to be particularly sensitive to the negative effects of radiation. ${ }^{39} 40$

It has recently been suggested that the lung clearance index (LCI), an index of uneven ventilation distribution measured by multiple breath 
washout (MBW) of an inert marker gas, could be a sensitive early marker of CF lung disease. ${ }^{13-15} 18$ The MBW method is not new but has been used for over 50 years, ${ }^{41}$ and paediatric studies were published during the $1960 \mathrm{~s}^{42}$ and 1970s. ${ }^{43}$ Breath-by-breath studies in children using modern PC technology were reported by $\mathrm{Wall}^{14}$ and $\mathrm{Kraemer}^{45}$ more than 20 years ago. LCI has advantages over spirometry in that it can be measured easily in all age groups and normative values are similar from early childhood to adulthood. ${ }^{15}$ It discriminates between patients with CF and healthy subjects better than either $\mathrm{FEV}_{1}$ or body plethysmography (residual volume/total lung capacity ratio). ${ }^{15}$ The LCI has also been shown to be an earlier and stronger indicator of disease progression than spirometry. ${ }^{46}$ Finally, the MBW test is a non-invasive functional measurement which involves no risk or hazardous exposures. The relationship of LCI to structural lung abnormalities in CF has, however, not been reported previously.

The present study aimed to assess the correlations between LCI and spirometry and structural lung abnormalities in CF, and to determine their sensitivity and specificity in the detection of bronchiectasis and other structural lung abnormalities as measured by HRCT. It was hypothesised that LCI would agree better with the presence of HRCT abnormalities than spirometry.

\section{METHODS}

\section{Subjects}

A retrospective study of spirometry, MBW and HRCT recordings was performed over a 30 -month period in 44 consecutive patients with CF aged $<20$ years. All patients underwent these investigations as part of their routine annual review at the Göteborg CF centre where HRCT is performed routinely every third year in patients over 5 years of age. Chronic colonisation with Pseudomonas aeruginosa was defined as three or more positive cultures over a 6 -month period. This retrospective analysis was approved by the ethics committee for human research at the University of Göteborg.

\section{Measurement of lung structure}

Lung structure was evaluated using HRCT scans. The scanning protocol has previously been reported ${ }^{24}$ and is described in more

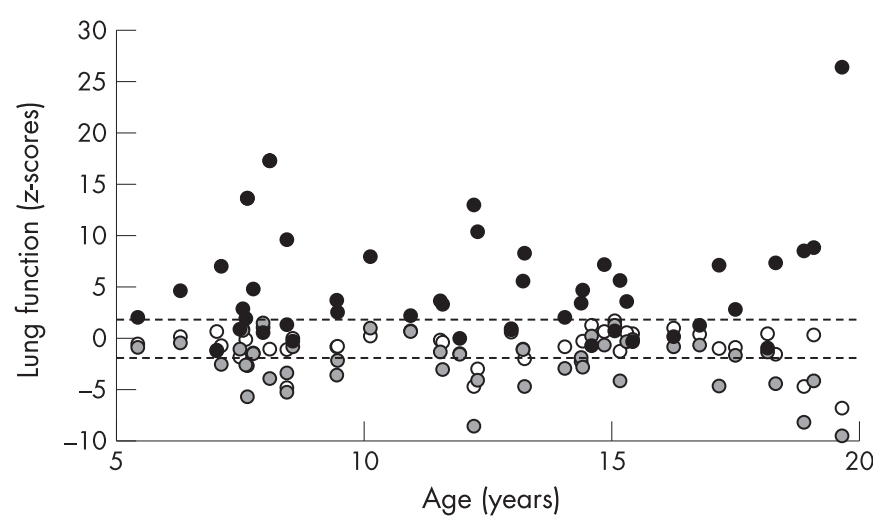

Figure 1 Lung function (lung clearance index (LCI), forced expiratory volume in $1 \mathrm{~s}\left(\mathrm{FEV}_{1}\right)$ and maximal expiratory flow when $75 \%$ of forced vital capacity was expired $\left.\left(\mathrm{FEF}_{75}\right)\right)$ expressed as z-scores plotted against age in 44 children and teenagers with CF. Black filled circles denote $\mathrm{LCl}$, open circles $\mathrm{FEV}_{1}$ and grey circles $\mathrm{FEF}_{75}$. The horizontal hatched lines denote the upper and lower limits of normality (LLN) for the lung function variables. detail in the online supplement. Briefly, images were obtained from lung apex to base at $15 \mathrm{~mm}$ (children) and $10 \mathrm{~mm}$ (adults $\geqslant 18$ years) intervals using $1.25 \mathrm{~mm}$ thick slices in inspiration. In addition, three expiratory HRCT images were obtained through the upper, middle and lower lung zones.

All scans were blinded to date and patient identification and were scored in random order by an experienced observer ${ }^{21} 2425$ using an adapted scoring system developed by Brody et al. ${ }^{22}{ }^{47}$ The scoring system evaluates the severity and extent of central and peripheral bronchiectasis; extent of central and peripheral mucus plugging; severity and extent of central and peripheral airway wall thickening; parenchymal abnormalities (extent of opacities, ground glass pattern and cysts and bullae); and gas trapping. Composite HRCT score and component HRCT scores were expressed as a percentage of the maximal score. ${ }^{24}{ }^{47}$

A composite CT score $>5 \%$ was set as abnormal based on findings in 15 normal individuals that were scored intermixed with patients with CF. All normal individuals had a CT score below 5\% (unpublished data). Air trapping involving more than $30 \%$ of the lung was also defined as abnormal. It is known that air trapping can be seen in normal individuals, ${ }^{48}$ and in other studies in the bronchiolitis obliterans syndrome a $32 \%$ cut-off has been used. ${ }^{49}$

\section{Measurement of lung function}

The lung function measurements are described in detail in the online supplement. Briefly, spirometric measurements included $\mathrm{FEV}_{1}$, forced vital capacity (FVC) and the maximal expiratory flow when $75 \%$ of $\mathrm{FVC}$ was expired $\left(\mathrm{FEF}_{75}\right)$. They were related to normative values for Swedish children $\left(7-18\right.$ years) ${ }^{50}$ or adults. ${ }^{51} 52$ The lower limit of normality (LLN) was defined as the predicted mean minus 1.96 residual standard deviations (RSD). In addition, tidal breathing multiple-breath sulfur hexafluoride $\left(\mathrm{SF}_{6}\right)$ washout was performed using a mass spectrometer for gas analysis, as previously described in detail elsewhere. ${ }^{15}$ The LCI was calculated as the number of lung volume turnovers (ie, the cumulative expired volume divided by the functional residual capacity) needed to lower the end-tidal tracer gas concentration to $1 / 40$ th of the starting concentration. ${ }^{15}$ A high value of LCI thus indicates abnormal ventilation distribution. The mean LCI result from three MBWs in each subject was used for analysis. In a previous study including healthy subjects, the mean, RSD and upper limit of normality (ULN; mean plus 1.96 RSD) for LCI were 6.33, 0.43 and 7.17, respectively. ${ }^{15}$

\section{Statistical analysis}

Abnormal structure was defined as a composite HRCT score of $>5 \%$, the presence of bronchiectasis or air trapping $>30 \%$. Lung function was expressed as z-scores which were calculated as (measured value - predicted value)/RSD from the reference population. Abnormal lung function was defined as LCI above +1.96 z-scores or $\mathrm{FEV}_{1}$ or $\mathrm{FEF}_{75}$ below -1.96 z-scores. The proportions of patients with normal or abnormal $\mathrm{FEV}_{1}$ or LCI results in relation to HRCT classifications (cross tabulations) were compared using the Yates corrected $\chi^{2}$ test. The sensitivity and specificity were determined for LCI, FEV 1 and $\mathrm{FEF}_{75}$ with respect to an abnormal HRCT composite score, the presence of bronchiectasis and the presence of abnormal air trapping, as diagnosed by HRCT scanning. Sensitivity was calculated as the proportion of the study population with abnormal HRCT findings that showed abnormal lung function results. Specificity was calculated as the proportion of the study population with 


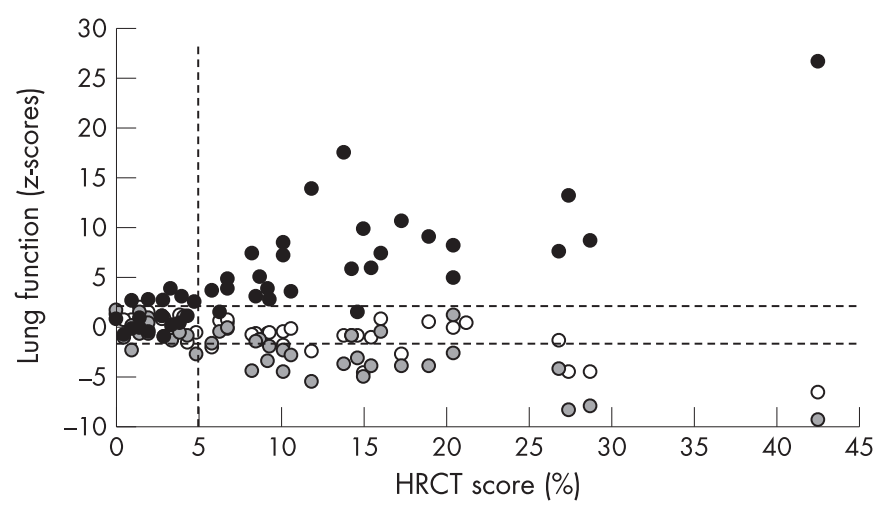

Figure 2 Lung function (lung clearance index (LCI), forced expiratory volume in $1 \mathrm{~s}\left(\mathrm{FEV}_{1}\right)$ and maximal expiratory flow when $75 \%$ of forced vital capacity was expired $\left.\left(\mathrm{FEF}_{75}\right)\right)$ expressed as z-scores plotted against HRCT (composite scores) in 44 children and teenagers with CF. Black filled circles denote $\mathrm{LCl}$, open circles $\mathrm{FEV}_{1}$ and grey circles $\mathrm{FEF}_{75}$. Vertical hatched line denotes upper limit of normality (ULN) for HRCT score and horizontal hatched lines denote upper and lower limits of normality (LLN) for the lung function variables.

normal HRCT finding that had normal lung function findings. The $95 \%$ confidence intervals (CI) around the sensitivity and specificity findings were calculated as follows: $95 \%$ CI for $\mathrm{p}=\mathrm{p} \pm 1.96^{*}$ SE; SE for $\mathrm{p}=\left(\mathrm{p}^{*}(1-\mathrm{p}) / \mathrm{n}\right)^{1 / 2}$ where $\mathrm{p}$ denotes the sensitivity or specificity expressed as a ratio.

Spearman rank correlation coefficients $\left(R_{s}\right)$ were calculated for $\mathrm{FEV}_{1}$, LCI and $\mathrm{FEF}_{75}$ with respect to the HRCT composite score, bronchiectasis, mucus plugging, airway wall thickness, parenchyma and air trapping. A p value of $<0.05$ was accepted as statistically significant. Statistica 6.0 (StatSoft, Tulsa, Oklahoma, USA) was used for the statistical analyses.

\section{RESULTS}

The study group included 27 males and 17 females aged 5.419.6 years (mean and median age 12.2 years). Twenty-one patients (48\%) were homozygotes and $19(43 \%)$ were heterozygotes for dF508. Eleven patients were chronically colonised with Pseudomonas aeruginosa and 37 were pancreatic insufficient.
$\mathrm{FEV}_{1}$ ranged from $44 \%$ to $127 \%$ of predicted values (mean and median 95\%).

Bronchiectasis was diagnosed in 26 patients (59\%), an abnormal composite HRCT score $(>5 \%)$ was found in 27 patients (61\%), and pathological gas trapping was present in 16 $(36 \%)$. Figure 1 gives lung function findings in $z$-scores versus age. Age was evenly distributed and there was no significant correlation between any lung function parameter and age. Figure 2 shows lung function in relation to HRCT scores. Of the 27 patients with an HRCT score $>5 \%, 25$ had an abnormally elevated LCI, 17 had abnormal $\mathrm{FEF}_{75}$ results and 7 had reduced $\mathrm{FEV}_{1}$. Seventeen patients had normal HRCT scores $(\leqslant 5 \%) ; 6$ of these had elevated LCI, 2 had abnormal $\mathrm{FEF}_{75}$ and all had $\mathrm{FEV}_{1}$ within normal limits.

LCI and $\mathrm{FEF}_{75}$ showed closer agreement with findings of structural lung abnormalities than did $\mathrm{FEV}_{1}$ (table 1). The LCI had the best sensitivity (85-94\%) for detecting structural lung abnormalities (bronchiectasis, composite HTCT score and air trapping) (table 2). The sensitivity for $\mathrm{FEV}_{1}$ ranged from $19 \%$ to $26 \%$ and for $\mathrm{FEF}_{75}$ from $62 \%$ to $75 \%$. The specificity in the detection of structural lung disease was, however, higher for $\mathrm{FEV}_{1}(89-100 \%)$ and $\mathrm{FEF}_{75}$ (75-88\%) than for LCI (43-65\%).

The LCI had a markedly stronger correlation with all six structural abnormality scores $\left(R_{s} 0.65\right.$ to 0.85$)$ than did the $F V_{1}$ $\left(\mathrm{R}_{\mathrm{s}}-0.35\right.$ to -0.62$)$ or $\mathrm{FEF}_{75}\left(\mathrm{R}_{\mathrm{s}}-0.44\right.$ to -0.66$)$ (table 3$)$.

The proportion of patients with normal or abnormal $\mathrm{FEF}_{75}$ showed better agreement with LCI classification than did the $\mathrm{FEV}_{1}$ (table 4), but the overall correlation with LCI was similar $\left(\mathrm{FEF}_{75}: \mathrm{R}_{\mathrm{s}}=-0.66, \mathrm{p}<0.001 ; \mathrm{FEV}_{1}: \mathrm{R}_{\mathrm{s}}=-0.63, \mathrm{p}<0.001\right)$.

\section{DISCUSSION}

This study aimed to determine the sensitivity of spirometry and MBW for detecting bronchiectasis and other structural lung changes as measured by HRCT scanning in children and teenagers with $\mathrm{CF}$, and to compare the correlations between LCI and spirometry and structural lung abnormalities. The hypothesis was that the LCI would be a more sensitive test for detecting structural lung disease than $\mathrm{FEV}_{1}$ or $\mathrm{FEF}_{75}$, and that LCI would correlate better than spirometry with structural lung changes.

Table 1 Agreement between $\mathrm{LCl}_{1} \mathrm{FEV}_{1}$ and $\mathrm{FEF}_{75}$ and structural lung changes classified as abnormal or normal

\begin{tabular}{|c|c|c|c|c|c|c|}
\hline & \multicolumn{2}{|c|}{ Bronchiectasis } & \multicolumn{2}{|c|}{ HRCT score } & \multicolumn{2}{|c|}{ Air trapping } \\
\hline & Yes & No & $>5 \%$ & $\leqslant 5 \%$ & $>\mathbf{3 0} \%$ & $\leqslant \mathbf{3 0} \%$ \\
\hline \multicolumn{7}{|l|}{ LCI } \\
\hline Abnormal & 22 & 9 & 25 & 6 & 15 & 16 \\
\hline \multirow[t]{2}{*}{ Normal } & 4 & 9 & 2 & 11 & 1 & 12 \\
\hline & \multicolumn{2}{|c|}{$p=0.033$} & \multicolumn{2}{|c|}{$\mathrm{p}<0.001$} & \multicolumn{2}{|c|}{$p=0.027$} \\
\hline
\end{tabular}

FEV $_{1}$

$\begin{array}{lllllll}\text { Abnormal } & 5 & 2 & 7 & 0 & 4 & 3 \\ \text { Normal } & 21 & 16 & 20 & 17 & 12 & 25 \\ & p=0.761 & & p=0.062 & & p=0.413\end{array}$

$\mathrm{FEF}_{75}$

$\begin{array}{lllllll}\text { Abnormal } & 16 & 3 & 17 & 2 & 12 & 7 \\ \text { Normal } & 10 & 15 & 10 & 15 & 4 & 21 \\ & p=0.008 & & p=0.003 & & p=0.004 & \end{array}$

$\mathrm{LCl}$, lung clearance index; $\mathrm{FEV}_{1}$, forced expiratory volume in $1 \mathrm{~s} ; \mathrm{FEF}_{75}$, maximal expiratory flow when $75 \%$ of forced vital capacity was expired; HRCT, high-resolution CT.

HRCT score $>5 \%$ is defined as abnormal and $>30 \%$ air trapping is defined as abnormal.

$p$ Values refer to the Yates corrected $\chi^{2}$ test. 
Table 2 Sensitivity and specificity (expressed as percentage with 95\% confidence intervals in parentheses) for $\mathrm{LCl}_{1} \mathrm{FEV}_{1}$ and $\mathrm{FEF}_{75}$ with respect to HRCT-defined structural lung abnormalities

\begin{tabular}{|c|c|c|c|}
\hline & Bronchiectasis & HRCT score & Air trapping \\
\hline \multicolumn{4}{|l|}{$\mathrm{LCl}$} \\
\hline Sensitivity & 85 (71 to 98$)$ & 93 (83 to 100$)$ & 94 (82 to 100$)$ \\
\hline Specificity & 50 (27 to 73$)$ & 65 (42 to 87 ) & $43(25$ to 61$)$ \\
\hline \multicolumn{4}{|l|}{$\mathrm{FEV}_{1}$} \\
\hline Sensitivity & $19(4$ to 34$)$ & $26(9$ to 42$)$ & 25 (4 to 46$)$ \\
\hline Specificity & $89(74$ to 100$)$ & $100(100$ to 100$)$ & 89 (78 to 100$)$ \\
\hline \multicolumn{4}{|l|}{$\mathrm{FEF}_{75}$} \\
\hline Sensitivity & 62 (43 to 80 ) & 63 (45 to 81 ) & 75 (54 to 96$)$ \\
\hline Specificity & 83 (66 to 100$)$ & $88(73$ to 100$)$ & 75 (59 to 91$)$ \\
\hline
\end{tabular}

It was shown that LCI was markedly more sensitive than either $\mathrm{FEV}_{1}$ or $\mathrm{FEF}_{75}$ with respect to structural lung abnormalities (bronchiectasis, air trapping and combined abnormalities). The sensitivity of LCI ranged between $85 \%$ and $94 \%$. Of 27 patients with abnormal HRCT scores, 25 had elevated LCI and $22 / 26$ patients with evidence of bronchiectasis had abnormal LCI, which suggests that a normal LCI in a subject with CF almost excludes structural lung damage. The specificity of LCI was modest, however, because LCI was abnormal in onethird of the patients with normal HRCT scores, half of those without evidence of bronchiectasis, and in more than half of those without diagnosed air trapping.

The LCI is a measure of overall ventilation inhomogeneity in the lungs, which occurs at branch points in large or small airways including very small airways close to or within the gas exchange zone. ${ }^{53}$ Geometric alterations in very small airways due to airway wall thickening or mucus accumulation can result in uneven ventilation distribution, but such alterations may not be detected by HRCT scanning. The LCI may therefore be abnormally elevated in subjects with CF lung disease but lack HRCT evidence of structural lung changes. Another explanation for the somewhat low specificity of the LCI could be that our HRCT protocol might have missed some structural aberrations. Only three expiratory images were obtained, which may limit the ability to detect the full extent of air trapping. In addition, for the inspiratory CT scans there are relatively large gaps of $15 \mathrm{~mm}$ between the CT images, which potentially result in underestimation of the structural abnormalities. ${ }^{36}$ It has recently been shown that bronchiectasis can be missed using an incremental HRCT protocol compared with a full lung HRCT scan. ${ }^{36}{ }^{54}$ Conventional HRCT scanning is therefore no longer the ideal method and is now often replaced by full lung CT scans.

We set an abnormal composite CT score as a score of $>5 \%$. In 15 normal individuals that were scored intermixed with patients with CF, all normal individuals had a CT score of $<5 \%$ (unpublished data). We defined an abnormal amount of air trapping as involving $>30 \%$ of the lung. It is known that air trapping can be seen in normal individuals, ${ }^{48}$ and in other studies in the bronchiolitis obliterans syndrome a $32 \%$ cut-off has been used. ${ }^{49}$

As expected, the $\mathrm{FEV}_{1}$ had a low sensitivity, making it of little value in early disease detection in CF. This corresponds to previous studies showing structural disease in about $30 \%$ of patients with normal spirometric parameters. ${ }^{21} 2425$ The better specificity of $\mathrm{FEV}_{1}$ compared with the LCI means that, when $\mathrm{FEV}_{1}$ starts to decline, major bronchiectasis or other structural damage is likely to be present. On the other hand, many patients in the present study who had normal $\mathrm{FEV}_{1}$ or $\mathrm{FEF}_{75}$ results showed evidence of quite advanced structural lung damage. The present study suggests that the $\mathrm{FEF}_{75}$ is considerably more sensitive than $\mathrm{FEV}_{1}$ for detecting structural alterations. This reflects the closer agreement shown between abnormal LCI and $\mathrm{FEF}_{75}$ than $\mathrm{FEV}_{1}$, and supports the common view that $\mathrm{FEF}_{75}$ is more sensitive than $\mathrm{FEV}_{1}$ to small airway disease. Nevertheless, the high frequency of abnormal LCI in CF and the strong correlation between LCI and HRCT abnormalities demonstrate that the LCI is a better indictor of structural lung abnormalities than spirometry.

Because this study was cross-sectional, we can only speculate that the LCI will rise in parallel with advancing structural lung alterations in patients with CF who are followed over several years. This important question needs to be addressed in future longitudinal studies. Based on the present findings, it may nevertheless be argued that the MBW test should be used to monitor lung disease in young children with CF. As long as the LCI remains normal, the presence of structural lung abnormalities is unlikely and HRCT scanning is probably of limited value to verify disease progression. Only when the LCI starts to rise may an HRCT scan be needed to confirm disease progression. This could be a dose-saving strategy of particular importance for young children who are more sensitive to radiation. Further investigations are needed, however, before firm clinical recommendations regarding such a strategy can be

Table 3 Correlation between $\mathrm{LCl}_{1} \mathrm{FEV}_{1}, \mathrm{FEF}_{75}$ (standard deviation scores) and structural abnormalities

\begin{tabular}{lcccccr}
\hline & HRCT score & Bronchiectasis & Mucus plugs & Airway wall thickness & Parenchyma & Air trapping \\
\hline $\mathrm{LCl}$ & $0.85^{* * *}$ & $0.64^{* * *}$ & $0.73^{* * *}$ & $0.76^{* * *}$ & $0.79^{* * *}$ & $0.65^{* * *}$ \\
$\mathrm{FEV}_{1}$ & $-0.62^{* * *}$ & $-0.35^{*}$ & $-0.45^{* *}$ & $-0.55^{* * *}$ & $-0.58^{* * *}$ & $-0.59^{* * *}$ \\
$\mathrm{FEF}_{75}$ & $-0.66^{* * *}$ & $-0.44^{* *}$ & $-0.49^{* * *}$ & $-0.56^{* * *}$ & $-0.65^{* * *}$ & $-0.57^{* * *}$ \\
\hline
\end{tabular}

$\mathrm{LCl}$, lung clearance index; $\mathrm{FEV}_{1}$, forced expiratory volume in $1 \mathrm{~s} ; \mathrm{FEF}_{75}$, maximal expiratory flow when $75 \%$ of forced vital capacity was expired; HRCT, high-resolution CT. Data shown as Spearman rank correlation coefficients $\left(R_{s}\right)$.

${ }^{*} \mathrm{p}<0.05 ;{ }^{*} \mathrm{p}<0.01 ;{ }^{* * *} \mathrm{p}<0.001$. 
Table 4 Agreement between LCl and spirometry classified as abnormal or normal

\begin{tabular}{|c|c|c|c|c|}
\hline & \multicolumn{2}{|l|}{ FEV $_{1}$} & \multicolumn{2}{|l|}{$\mathrm{FEF}_{75}$} \\
\hline & Abnormal & Normal & Abnormal & Normal \\
\hline \multicolumn{5}{|l|}{ LCl } \\
\hline Abnormal & 7 & 24 & 18 & 13 \\
\hline \multirow[t]{2}{*}{ Normal } & 0 & 13 & 1 & 12 \\
\hline & \multicolumn{2}{|l|}{$p=0.065$} & \multicolumn{2}{|l|}{$p=0.006$} \\
\hline
\end{tabular}

$\mathrm{LCl}$, lung clearance index; $\mathrm{FEV}_{1}$, forced expiratory volume in $1 \mathrm{~s} ; \mathrm{FEF}_{75}$, maximal expiratory flow when $75 \%$ of forced vital capacity was expired.

$p$ Values refer to the Yates corrected $\chi^{2}$ test.

given. It is acknowledged that both HRCT and LCI should be viewed as potential surrogate outcome markers in CF at this time. Their relationships to the long-term outcome of CF lung disease-as reflected in widely accepted outcome markers such as $\mathrm{FEV}_{1}$ or mortality - are not fully known. Nevertheless, when an aggressive approach to early CF lung disease is evaluated, more sensitive outcomes than $\mathrm{FEV}_{1}$ and mortality are definitely needed.

It is unlikely that a bias has influenced the general outcome of the study. The MBW parameters were determined without having information on the CT scan. Furthermore, patient identity, demographic information or lung function findings were not available to the observer scoring the images. Ideally, spirometry, MBW and HRCT scans should have been performed on the same day in a randomised fashion. All investigations were carried out in clinically stable patients during their annual review at the CF centre. They were undertaken on the same day in 26 of the patients (59\%), and in all remaining patients but one there was only one day between any of the tests. The patient group included a sample of approximately one-third of all patients with CF attending the CF Center in Göteborg. This sample constituted all consecutive patients over a 30-month period aged $<20$ years who were investigated with spirometry and MBW as part of their annual review and, in addition, HRCT which is done routinely every third year in patients older than 5 years. There was a slight over-representation of males $(61 \%)$ in the study population compared with $52 \%$ in the entire CF population. The study involved only children and teenagers who could perform spirometric tests and who were aged $>5$ years because the routine schedule includes HRCT scans only above that age. The relationships between LCI and HRCT abnormalities in younger and older patients therefore remain to be determined.

In conclusion, this cross-sectional analysis shows that the LCI is more sensitive than spirometry $\left(\mathrm{FEV}_{1}\right.$ or $\left.\mathrm{FEF}_{75}\right)$ for detecting structural lung alterations in CF, and that a normal LCI indicates the absence of structural lung damage detectable by HRCT. The usefulness of LCI for detecting progression of CF lung disease compared with HRCT needs to be assessed in longitudinal studies.

Funding: Pim de Jong was supported by a British Columbia Lung Association Fellowship in Respiratory Medicine and a Canadian Institute of Health Research/ Michael Smith Foundation Transplant Research Training Award.

Competing interests: None.

\section{REFERENCES}

1. Gibson RL, Burns JL, Ramsey BW. Pathophysiology and management of pulmonary infections in cystic fibrosis. Am J Respir Crit Care Med 2003;168:918-51.

2. Tiddens HA. Detecting early structural lung damage in cystic fibrosis. Pediatr Pulmonol 2002;34:228-31.

3. Nir M, Lanng S, Johansen HK, et al. Long-term survival and nutritional data in patients with cystic fibrosis treated in a Danish centre. Thorax 1996;51:1023-7.
4. Stern RC. Denmark to the rescue. Pediatr Pulmonol 1996;21:151-2.

5. Frederiksen B, Lanng S, Koch C, et al. Improved survival in the Danish centertreated cystic fibrosis patients: results of aggressive treatment. Pediatr Pulmonol 1996;21:153-8.

6. Brody AS, Tiddens HA, Castile RG, et al. Computed tomography in the evaluation of cystic fibrosis lung disease. Am J Respir Crit Care Med 2005;172:1246-52.

7. Aurora $\mathbf{P}$, Wade $A$, Whitmore $\mathbf{P}$, et al. A model for predicting life expectancy of children with cystic fibrosis. Eur Respir J 2000;16:1056-60.

8. Hayllar KM, Williams SG, Wise AE, et al. A prognostic model for the prediction of survival in cystic fibrosis. Thorax 1997;52:313-7.

9. Sharma R, Florea VG, Bolger AP, et al. Wasting as an independent predictor of mortality in patients with cystic fibrosis. Thorax 2001;56:746-50.

10. Tauber $\mathbf{E}$, Eichler I, Gartner $\mathrm{C}$, et al. Improvements of lung function in cystic fibrosis. Pediatr Pulmonol 2002;33:263-8.

11. Corey M, Levison $\mathrm{H}$, Crozier $\mathrm{H}$. Five- to seven-year course of pulmonary function in cystic fibrosis. Am Rev Respir Dis 1976;114:1085-92.

12. Farrell PM, Li Z, Kosorok MR, et al. Longitudinal evaluation of bronchopulmonary disease in children with cystic fibrosis. Pediatr Pulmonol 2003;36:230-40.

13. Aurora P, Bush A, Gustafsson P, et al. Multiple-breath washout as a marker of lung disease in preschool children with cystic fibrosis. Am J Respir Crit Care Med 2005; 171:249-56.

14. Aurora $\mathbf{P}$, Gustafsson $\mathrm{P}$, Bush A, et al. Multiple breath inert gas washout as a measure of ventilation distribution in children with cystic fibrosis. Thorax 2004;59:1068-73.

15. Gustafsson PM, Aurora P, Lindblad A. Evaluation of ventilation maldistribution as an early indicator of lung disease in children with cystic fibrosis. Eur Respir $J$ 2003:22:972-9.

16. Eigen H, Bieler $\mathrm{H}$, Grant D, et al. Spirometric pulmonary function in healthy preschool children. Am J Respir Crit Care Med 2001;163:619-23.

17. Stocks J, Sly PD, Morris MG, et al. Standards for infant respiratory function testing: what(ever) next? Eur Respir J 2000;16:581-4.

18. Lum S, Gustafsson $\mathrm{P}$, Ljungberg $\mathrm{H}$, et al. Early detection of cystic fibrosis lung disease: multiple-breath washout vs. raised volume tests. Thorax 2007;62:341-7.

19. Robinson TE, Leung AN, Northway WH, et al. Composite spirometric-computed tomography outcome measure in early cystic fibrosis lung disease. Am J Respir Crit Care Med 2003;168:588-93

20. Long FR, Williams RS, Castile RG. Structural airway abnormalities in infants and young children with cystic fibrosis. J Pediatr 2004;144:154-61.

21. de Jong PA, Ottink MD, Robben SG, et al. Pulmonary disease assessment in cystic fibrosis: comparison of CT scoring systems and value of bronchial and arterial dimension measurements. Radiology 2004;231:434-9.

22. Brody AS, Klein JS, Molina PL, et al. High-resolution computed tomography in young patients with cystic fibrosis: distribution of abnormalities and correlation with pulmonary function tests. J Pediatr 2004;145:32-8.

23. Brody AS, Sucharew H, Campbell JD, et al. Computed tomography correlates with pulmonary exacerbations in children with cystic fibrosis. Am J Respir Crit Care Med 2005; 172:1128-32.

24. de Jong PA, Lindblad A, Rubin L, et al. Progression of lung disease on computed tomography and pulmonary function tests in children and adults with cystic fibrosis. Thorax 2006;61:80-5

25. de Jong PA, Nakano $\mathrm{Y}$, Lequin $\mathrm{MH}$, et al. Progressive damage on high resolution computed tomography despite stable lung function in cystic fibrosis. Eur Respir J 2004:23:93-7.

26. Kang EY, Miller RR, Muller NL. Bronchiectasis: comparison of preoperative thinsection CT and pathologic findings in resected specimens. Radiology 1995;195:64954.

27. Goris ML, Zhu HJ, Blankenberg F, et al. An automated approach to quantitative air trapping measurements in mild cystic fibrosis. Chest 2003;123:1655-63.

28. Bonnel AS, Song SM, Kesavarju K, et al. Quantitative air-trapping analysis in children with mild cystic fibrosis lung disease. Pediatr Pulmonol 2004;38:396-405.

29. Dodd JD, Barry SC, Barry RB, et al. Thin-section CT in patients with cystic fibrosis: correlation with peak exercise capacity and body mass index. Radiology 2006:240:236-45

30. Bhalla $\mathbf{M}$, Turcios $\mathbf{N}$, Aponte $\mathbf{V}$, et al. Cystic fibrosis: scoring system with thinsection CT. Radiology 1991;179:783-8.

31. Maffessanti M, Candusso M, Brizzi F, et al. Cystic fibrosis in children: HRCT findings and distribution of disease. J Thorac Imaging 1996;11:27-38.

32. Helbich TH, Heinz-Peer G, Eichler I, et al. Cystic fibrosis: CT assessment of lung involvement in children and adults. Radiology 1999;213:537-44.

33. Helbich TH, Heinz-Peer G, Fleischmann D, et al. Evolution of CT findings in patients with cystic fibrosis. AJR Am J Roentgenol 1999;173:81-8.

34. Santamaria F, Grillo G, Guidi G, et al. Cystic fibrosis: when should high-resolution computed tomography of the chest be obtained? Pediatrics 1998;101:908-13

35. de Jong PA, Mayo JR, Golmohammadi K, et al. Estimation of cancer mortality associated with repetitive computed tomography scanning. Am J Respir Crit Care Med 2006;173:199-203.

36. de Jong PA, Nakano $\mathrm{Y}$, Lequin $\mathrm{MH}$, et al. Dose reduction for $\mathrm{CT}$ in children with cystic fibrosis: is it feasible to reduce the number of images per scan? Pediatr Radiol 2006;36:50-3.

37. de Jong PA, Long FR, Nakano Y. Computed tomography dose and variability of airway dimension measurements: how low can we go? Pediatr Radiol 2006;36:1043-7. 
38. Long FR, Castile RG, Brody AS, et al. Lungs in infants and young children: improved thin-section CT with a noninvasive controlled-ventilation technique-initial experience. Radiology 1999;212:588-93.

39. Brenner D, Elliston C, Hall E, et al. Estimated risks of radiation-induced fatal cancer from pediatric CT. AJR Am J Roentgenol 2001;176:289-96.

40. Brenner DJ. Estimating cancer risks from pediatric CT: going from the qualitative to the quantitative. Pediatr Radiol 2002;32:228-34.

41. Becklake MR. A new index of the intrapulmonary mixture of inspired air. Thorax 1952: 7:111-6.

42. Kjellman B. Lung function in children with pneumonia. With special reference to distribution of inspired air and regional lung function. Scand J Respir Dis 1968;49:185-201.

43. Svenonius $\mathbf{E}$, Lecerof $H$, Lilja $B$, et al. The volume of trapped gas: a new and sensitive test for the detection of exercise-induced bronchospasm in children. Acta Paediatr Scand 1978:67:583-9.

44. Wall MA. Moment analysis of multibreath nitrogen washout in young children. J App/ Physiol 1985;59:274-9.

45. Kraemer R, Meister B. Fast real-time moment-ratio analysis of multibreath nitrogen washout in children. J Appl Physiol 1985;59:1137-44.

46. Kraemer R, Blum A, Schibler A, et al. Ventilation inhomogeneities in relation to standard lung function in patients with cystic fibrosis. Am J Respir Crit Care Med 2005;171:371-8.
47. Brody AS. Early morphologic changes in the lungs of asymptomatic infants and young children with cystic fibrosis. J Pediatr 2004;144:145-6.

48. Tanaka N, Matsumoto T, Miura G, et al. Air trapping at CT: high prevalence in asymptomatic subjects with normal pulmonary function. Radiology 2003;227:77685.

49. Bankier AA, Van Muylem A, Knoop C, et al. Bronchiolitis obliterans syndrome in heart-lung transplant recipients: diagnosis with expiratory CT. Radiology 2001;218:533-9.

50. Solymar L, Aronsson PH, Bake B, et al. Nitrogen single breath test, flow-volume curves and spirometry in healthy children, 7-18 years of age. Eur $\mathrm{J}$ Respir Dis 1980;61:275-86.

51. Hedenstrom H, Malmberg P, Fridriksson HV. Reference values for lung function tests in men: regression equations with smoking variables. Ups J Med Sci 1986;91:299310.

52. Hedenstrom $\mathbf{H}$, Malmberg $P$, Agarwal $K$. Reference values for lung function tests in females. Regression equations with smoking variables. Bull Eur Physiopathol Respir 1985;21:551-7.

53. Gustafsson P. Peripheral airway involvement in CF and asthma compared by inert gas washout. Pediatr Pulmonol 2007:42:168-76.

54. Dodd JD, Souza CA, Muller NL. Conventional high-resolution CT versus helical highresolution MDCT in the detection of bronchiectasis. AJR Am J Roentgenol 2006;187:414-20.

\section{Lung alert}

\section{A new treatment proposed for lung transplant rejection}

Despite intensive immunosuppressant therapy following lung transplantation, acute rejection occurs in over $50 \%$ of recipients within the first 6 months. Bronchiolitis obliterans syndrome (BOS) is related to acute rejection and independently contributes to mortality. It is thought that $\mathrm{T}$ cell depleting agents might reduce the incidence of these complications. Alemtuzumab is a humanised monoclonal antibody to the CD52 antigen which is expressed on T cells, B cells, monocytes, macrophages and platelets and causes lymphocyte depletion. The authors studied the effects of this agent on 12 patients with refractory acute rejection (RAR; ie, with biopsy proven rejection over at least 4 weeks) and 10 patients with BOS.

All patients had failed to respond to corticosteroids and antithymocyte globulin (ATG). The efficacy of alemtuzumab for the treatment of RAR was compared with ATG using the rejection grades on consecutive biopsies immediately before and after treatment. In patients with RAR, a significant and sustained reduction was observed in A and B grade biopsy rejection after alemtuzumab $(p<0.001)$. Treatment resulted in a significant reduction in the severity of RAR immediately, while there was no change in the mean severity after treatment with ATG. There was no significant change in mean forced expiratory volume in $1 \mathrm{~s}$ in BOS after treatment, but there was an improvement in the BOS scores. One-year graft survival for RAR after alemtuzumab treatment was $86 \%$. Survival with BOS at 1 and 2 years was $69 \%$.

Alemtuzumab is potentially useful in the treatment of RAR or BOS in lung transplant recipients who have failed prior conventional therapy, but this study is limited by the lack of a control arm. The authors point out that a randomised controlled study is required before recommending this treatment over existing therapies.

- Reams BD, Musselwhite LW, Zaas DW, et al. Alemtuzumab in the treatment of refractory acute rejection and bronchiolitis obliterans syndrome after human lung transplantation. Am J Transplant 2007;7:2802-8

\section{Ghassan El-Sayed}

Correspondence to: G El-Sayed, ST1 in Respiratory Medicine, Homerton University Hospital, London, UK; ghassanovii@yahoo.co. uk 